\title{
OPTIMUM CONSTRUCTION RESOURCE IN SPACE, TIME AND COST
}

\author{
Chin-Chih Kuo ${ }^{1} \quad$ Ren-Jye Dzeng ${ }^{2}$ \\ 1. Ph.D. Candidate, Department of Civil Engineering, National Chiao- \\ Tung University,ROC.e-mail:cckuo@rsea.gov.tw \\ 2. Associate Professor, Department of Civil Engineering, National Chiao- \\ Tung University, ROC.e-mail:rjdzeng@cc.nctu.edu.tw
}

\begin{abstract}
The article summarizes the overall idea of the study and presented the results of the first stage -- "simulation visualized of construction schedule". At present, we have corresponded and combined the CPM network diagram and the $3 \mathrm{D}$ object model for dynamic presentation. This kind of 4D dynamic diagram always simulates the 3D outlook at any point of time in the process of simulating project construction in the way of assembling blocks to inquire and obtain the planning of construction resources, status of use and performance of execution and can be displayed according to the change in the construction sequence. The results of the study provide an optimal location for describing the spatial information and resources required for building construction. In addition, this can increase the friendliness and accuracy of the CPM network diagram and can be used for reviewing the problems of hidden constructing conflicts and interface or as the visualized basic document for works like quantity calculation, structure calculation, piping construction and maintenance.
\end{abstract}

Keywords: spatial resource, time-cost trade off, geographic information system (GIS), genetic algorithms (GA), 4D dynamic diagram, 3D object model

\section{FOREWORD}

Though contractors are all aware of the significant influence of spatial resource on the construction cost and duration of projects [7], the conventional model of time-cost trade off has so far failed to regard the spatial resource as an influential factor. The main reason is that there are no models and documents for spatial information to be described. Space is originally a limited construction resource. Regarding the construction of building structure, the spatial information for construction shall at least include space available and required during the construction of each activity, constructing route, machine and tool location and moving scope, storage and lift space as well variations and limits in these spaces by time. These resources, in the process of a construction project, are characterized by dynamic variability, complexity, repeated use and non-storage. Either numerical attributes or statistic drawings are sufficient for complete expression. In the existing construction documents, spatial information can only be marked in a simplified way. for example, 5th $\mathrm{FL}$ or $\mathrm{H}=3 \mathrm{~m}$. This has only slightly help for the exploration on the relation among space/time/cost.

In the study of "optimum construction resource in space, time and cost", we intend to use the 4D dynamic description document $(3 \mathrm{D}$ model $+\mathrm{CPM}$ network diagram), the construction cost and the spatial information required for the construction as the basic data. Base on these information, we make use of GIS's unique capability of describing and processing spatial data to attribute spatial information [4] through the classification, setting up and expression of attributes of construction space. Then, we will further use the fitness of the GA suitable for seeking the near global optimum of numerous fields [1]. The factors of space and time and cost will be expressed in the form of objective function, fitness function, constraints or parameters to obtain the optimum of the three.

The "simulation visualized of construction schedule" in this presentation is to present a 4D dynamic description document, which is the result of the first step presented in the overall study plan. This will provide a location for describing spatial information for construction and serve as the basic document for the study of spatial optimization. 


\section{BACKGROUND AND MOTIVATION}

The CPM network diagram is most commonly used in the scheduling and resource control of building construction and one of core tools for construction project management [2]. However, the friendliness and accuracy of the existing CPM are not optimal. Because it fails to express spatial states like the scope or location of each activity and the relative location with other activities, it is difficult for construction planners to accurately imagine and describe the outlook and available spaces of the field during the construction of each activity. This may result in severe difference between scheduling and planning and the actual construction situation. The arrangement of space and time in the field of each construction team may also generate perceptive difference and result in disputes. In addition, project progress is mostly indicated by the amount or percentage of completion or the construction location and lacks images for description of current situations. Hence, it is difficult for the remote administrative unit to form a concrete idea of the field progress.

It is far less effective and accurate to express project progress, current situations and spatial states with CPM data than with joint description in graphics. A construction project originally needs to be expressed in images and describe the ideas of design or construction because images have always been recognized as the optimal communicative interface and expressive tool. A CAD drawing file is the most commonly used expressive tool for images of construction projects. However, the existing 2D/3D statistic drawings can not present the outlook during construction, as they do not possess time concepts. Attribute database files are also very insufficient and not easy to use. Thus, they can not be used for describing the demand and supply as well as variations of construction resources and spatial information. Telling from the effects presented by each, both CPM and statistic drawings are insufficient for describing the spatial states of construction field and serve as the documents for planning and communication of construction progress. These descriptive limits can be overcome by linking the information of the two and presenting them.

\section{OBJECTIVE}

The study is to describe the activity content of CPM in the 3D model and the time concepts originally possessed by CPM as well as the capability of describing construction resource planning, use and executive performance. The purpose is to integrate the data of the two into a single system to become a 4D dynamic description document with attribute data, image data and time concepts. Thus, the spatial states of activities can be accurately described to serve as the prerequisite document for the study of spatial optimization and simulate the outlook presented in the project construction. In addition, it can inquire and obtain the use and executive performance of construction resources under this outlook to become an optimal visualized communication document.

\section{PROBLEM AND DIFFICULTY}

The problem in the study is that there is no overlapped data between the existing CAD drawing files and the CPM network diagram and thus provides no basis for linkage. The study encounters two difficulties. One is that several activities are under construction at the same time but their start/finish time is different and how to accurately determine the variations of several activities under construction. The second difficulty to be overcome in the stage of study is how to link and make dynamic presentation.

\section{REVIEW}

Zouein and Tommelein (1994), had developed a "MoveSchedule" model, it establishes a time-space trade-off relationship for each activity in the schedule. Its space conflict resolve dynamically introduces changes to activity schedule to the meet demand for space on the site over problematic time interval [7]. Riley and Tommelein (1996), had developed a "MovePlan" tool, it combined off-theshelf CAD and scheduling software to develop space plan that effort helped to identify spatial conflicts before they occurred in the field [8]. Sadonio and Tommelein and Zabelle (1998), had integrated database system with $\mathrm{CAD}$ to support the last designer focuses on materals sourcing and procurement [9]. Feng and Liu (2000), had presented a hybrid approach that combines simulation techniques and GA to solve the time-cost trade-off problem under uncertainty [3]. Jia (2000), had integrated KBES (Knowledge-Based Expert Systems) with GIS to represents and reasons about spatial knowledge and how it provides graphical display capabilities for representing spatial information and derived solutions [5]. Above research had sketched the path to solve the problem of optimal of construction spatial. But, they described spatial information was limited by numerical or 2D's mathematical shape only, that can not represent completely and accurately according to characters of construction spatial information. The main crux, they all short the 4D dynamic description document help to describe. 


\section{PROCEDURE}

The study uses the construction of a building structure as a case for demonstration. It is to present how to use a CPM progress network diagram made by $\mathrm{P} 3$ and a $2 \mathrm{D}$ construction drawing as the basic data, link the corresponding attribute and image files by the package of Schedule Simulator via the parametric 3D object model made by the package software Triforma of MicroStation, and establish the model of a 4D dynamic diagram on the platform of windows 98.

\section{6-1 Process Flow Chart of 4D Dynamic Description} Document

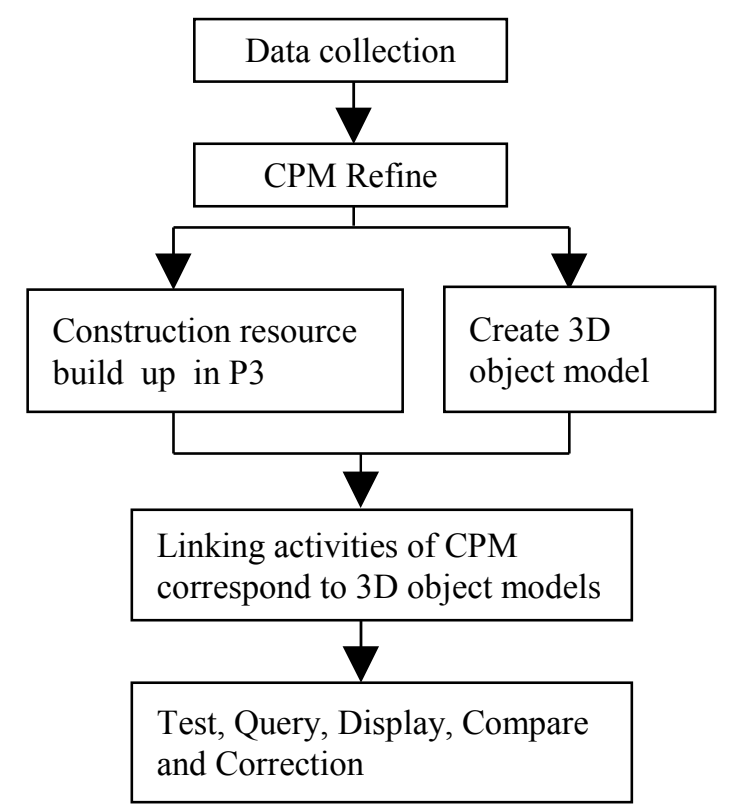

\section{6-2 Description of Key Points}

1. Determine the content, size, scope and shape of a block by the activity of CPM. Eliminate activities unnecessary or unsuitable for image representation, such as steel, formwork and trivial items (the number of activities was eliminated from 553 to 160 in demostraction case).

2. Input the planning and allocation states of construction resources like man, machine, material, time, cost and performance criteria information like BCWS (Budget Cost of Work Schedule), BCWP (Budget Cost of Work Performance) and ACWP (Actual Cost of Work Performance) into the activities of CPM.

3. Convert each structure element like column, beam, slab and wall into a parameteric 3D object drawing file called "part" in scale.

4. Make 3D blocks based on the activity content of CPM and combine several parts into a layer. A 3D block diagram consists of several layers and the image displayed is a block. Each activity corresponds to one or several 3D block diagrams (160 activities correspond to 184 blocks in demostraction case).

\section{RESULT AND CONTRIBUTION}

The results in the stage of the study are to provide a model for building a linkage between a CPM network diagram and a 3D model diagram to successfully generate a 4D dynamic diagram. The distinctive difference between the result drawing and the 3D model is that there is an additional time display window at the upper left (shown as appendix). The time window is the key result in the stage of the study. Users can freely change this time observation point. The Schedule Simulator uses the start/finish time in early/later as the basis for drawing file search to search those activities that are completed and under construction as well as the blocks of 3D model diagram corresponding to them. The Schedule Simulator generates a result diagram like piling up blocks by using CPM data as progress identification and giving difference color to present the outlook in construction in a visualized simulation way and displays the change in construction sequence accordingly.

In this stage of the study, the CPM software is used as the tool for store, search and retrieve of attribute data. Resource locate and executive performance can be inquired and obtained in a reverse way from a 4D dynamic diagram at any point of time and resource consumption can be inquired and obtained after these are added into the construction record.

The results in the stage of the study make three contributions to construction projects. First, they provide presenting points using each activity or time of a CPM network diagram, where users can inquire and obtain the 3D features of part of stimulation structure completed as well as planning, use and executive performance of construction resources. Thus, it not only can help construction workers have more concrete understanding of targets and performance and basis for discussing work situations, but also provides an optimal visualized communication tool among construction teams.

Second, users who can already familiar with such 4D dynamic description documents can have concrete understanding of previously hidden construction conflicts and eliminate them in advance to enable the construction schedule planning proposal to be closer to actual executions.

Third, a 4D dynamic description document provides an optimal location for describing spatial information and serves as a basic document for the study of spatial optimization. A 3D model diagram can serve as a visualized basic document for works 
like quantity calculation, structure calculation, piping construction and maintenance.

\section{CONCLUSIONS}

1. The study adopts a reference drawing for drawing display to enable the drawing file size to shrink from $20 \mathrm{M}$ byte to $400 \mathrm{~K}$ byte and achieve the purpose of rapid presentation.

2. Several activities are under construction at the same time but their start/finish time is different. Through CPM adjudge the 4D dynamic drawing can accurately control the parts that should be displayed by time while a 3D model diagram only allows manual approximate determination.

3. To use this visualized simulation model requires considerable memory capacity (RAM above $128 \mathrm{M}$ ), a high-end VGA card (above 16M, and supportive to Open GL) and CPU above 500MHZ.

4. To consider the convenience of obtaining software tools and the openness of data, MS-PROJECT and ACCESS can be used in place of the P3 and Schedule Simulator software tools adopted in the study and link via windows ODBC to achieve similar effects. However, the access of construction resource attribute files and dynamic presentation will not be so advantageous.

\section{CONSTRAINTS}

To use the model in the study requires making of a $3 \mathrm{D}$ object model corresponding to the activities of CPM. It is better not to change the construction scope included in the activities, otherwise it is necessary to re-modify the $3 \mathrm{D}$ model, which is equivalent to remaking of a new case. This is the most severe constraint of the results in the study.

Another constraint of the results in this stage of the study is that it is necessary to inquire and obtain simulated images through the media of the Schedule Simulator with time as the inquiry condition instead of direct access to the corresponding 3D model from the activities of CPM progress network diagram. This is not so optimal for users.

\section{SUGGESTION AND FUTURE}

The traditional 2D diagram, due to its limited capabilities of expression and description, often hide some drawing conflicts that can not be easily detected. On the contrary, due to easier view, the 3D design can discover and eliminate drawing errors or conflicts early. It is suggested that the $3 \mathrm{D}$ model is completed in the design stage and can be very helpful for the communication between designers and clients as well as subsequent works. This kind of 3D model made in scale has possessed location coordinates and sizes of structure elements. Future studies can use these data to directly transform to the execution of structure calculation software. Or they can use the volume, size and surface area data of these structure elements to easily complete quantity calculation for several items (such as concrete, finishing, painting, tiles, bricks etc.). Or they can use the data to be overlapped with the 3D data of piping in the same scale to examine the conflicts of piping construction.

The limit that the construction scope can not be changed can be avoided by adopting the linkage between one activity and several "parts". However, errors can easily occur, as the linkage is excessively complex and time-consuming. Nevertheless, at least it is not necessary to remake the expensive $3 \mathrm{D}$ model when the construction scope is changed. In addition, it can better fit into user habits by directly inquiring and obtaining the 3D model from the activities of CPM. This is the suggestion to those who are interested in construction visualization.

The study in future will continue to work on the descriptive model of construction spatial information and the study of spatial resource optimization based on the 4D dynamic description document.

\section{REFERENCES}

[1] Adeli, H., and S. L. Hung, Machine Learn-Neural Networks, Genetic Algorithms, and Fuzzy Systems, John-Wiley \& Sons, Inc., USA, 1995.

[2] Ahuja, Hira N., S. P. Dozzi, and S. M. Abourizk, Project Management: Techniques in Planning and Controlling Construction Projects, John-Wiley \& Sons, Inc., USA, 1994.

[3] Feng, Chung-Wei, and Liang Liu, and Scott a. Burns, "Stochastic Construction Time-Cost TradeOff Analysis", J. of Computing in Civil Engineering, ASCE, Vol. 14, No. 2, pp. 117 126, April 2000.

[4] Fotheringham, Stewart, and Peter Rogerson, Spatial Analysis and GIS, Taylor \& Francis Inc., USA, 1994.

[5] Jia, Xudong, "IntelliGIS: Tool for Representing and Reasoning Spatial Knowledge", J. of Computing in Civil Engineering, ASCE, Vol. 14, No. 1, pp. 51 59, Jan. 2000.

[6] Langran, Gail, Time in Geographic Information Systems, Taylor \& Francis Ltd., UK, 1992.

[7] Zouein, Pierrette P., and Iris D. Tommelein, "Time-Space Tradeoff Strategies for Space-Schedule Construction", Proceeding of the 1st Congress on Computing in Civil Engineer, ASCE, pp. 1172 1179, Washington, DC, USA, June 20 22 1994. 
[8] Riley, David R., and Iris D. Tommelein, "Space Planning Tools for Multi-Story Construction", Proceeding of the 3rd Congress on Computing in Civil Engineer, ASCE, pp. 718 724, 1996.

[9] Sadonio, Marcelo, Iris D. Tommelein, and Todd R. Zabelle, "Last Designer's CAD -- database for sourcing, procurement, and planning ", Proceedings of the 1998 International Computing Congress on Computing in Civil Engineering, ASCE, pp.364 375, Boston, MA, USA, Oct. 18 21, 1998.

[10] Tavakoli, A., and K. L. Klika, "Construction Management with AutoCAD", J. of Management in Engineering, Vol. 7, No. 3, pp. 267 278, 1991.

\section{APPENDIX}

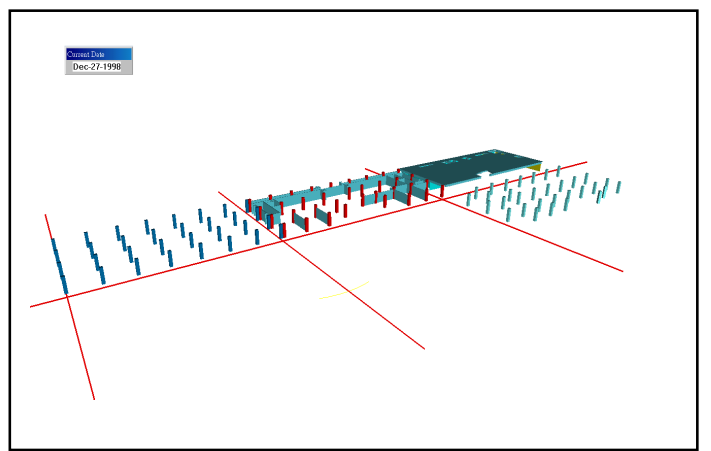

Figure 1. Status on Dec-27-1998

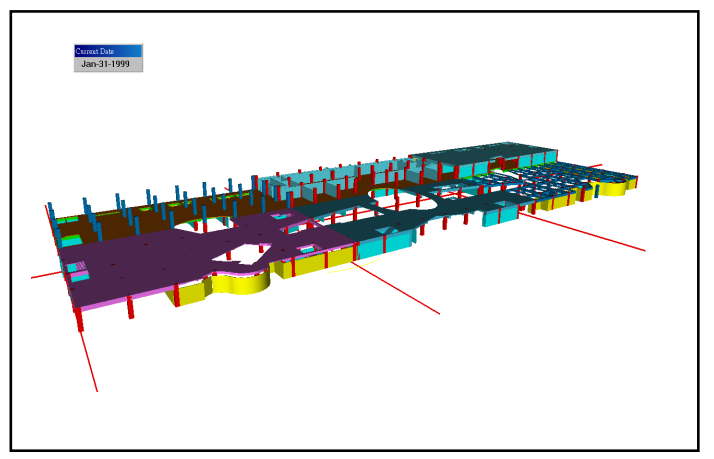


Figure 3. Status on Mar-28-1999

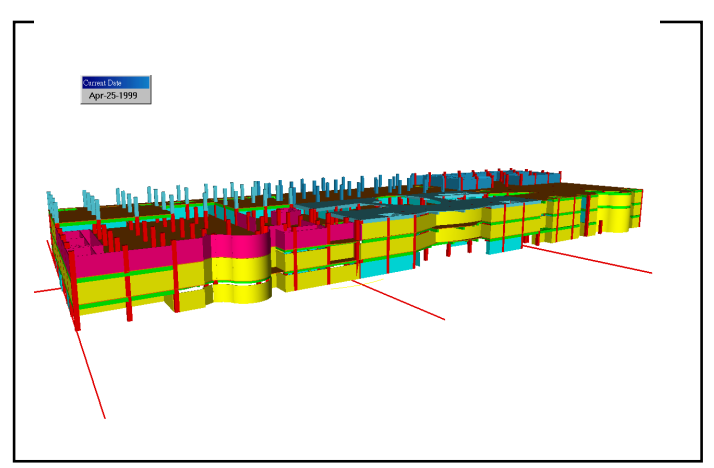

Figure 4. Status on Apr-25-1999

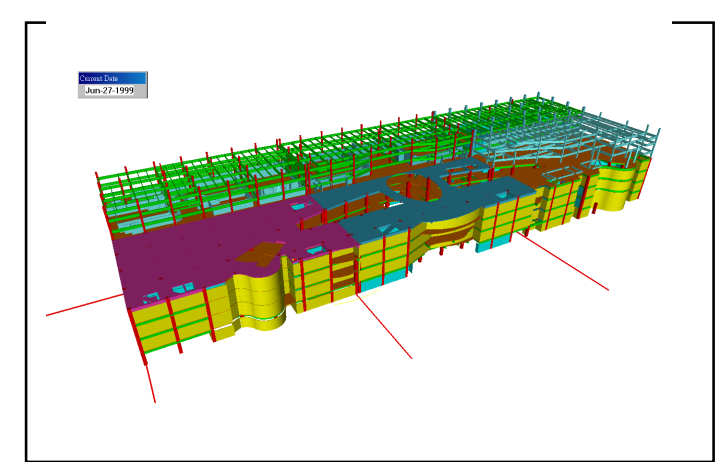

Figure 5. Status on Jun-27-1999

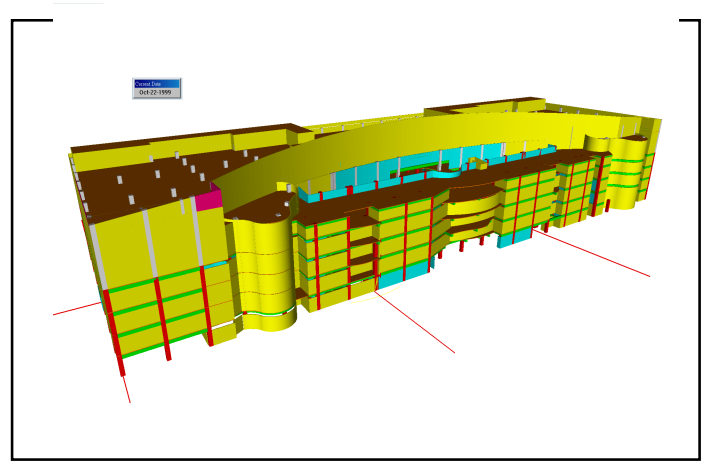

Figure 6. Status on Oct-22-1999 